\title{
The Capacity of the Fourth Chromosome of Drosophila melanogaster to Establish End-to-End Contacts with the Other Chromosomes in Salivary-Gland Cells*
}

\author{
Berwind P. Kaufmann and Helen Gay \\ Department of Zoology and Cytogenetics Laboratory of Carnegie Institution of \\ Washington \\ The University of Michigan, Ann Arbor, Michigan
}

Received January 31, 1969

\begin{abstract}
When the fourth chromosome of Drosophila melanogaster is attached, as the result of an induced translocation, to $21 \mathrm{~A}$ in $2 \mathrm{~L}$ or $60 \mathrm{E}$ in $2 \mathrm{R}$, its tip exhibits a marked capacity to pair end-to-end with the tips of the other chromosomes. In each of the translocations, about 59 per cent of the contacts involving the tip of 4 were with the tip of $X$. If this pairing preference reflects structural similarity, tho tip of 4 is much more like the tip of $\mathrm{X}$ than that of any other chromosome. The significance of this phenomenon is discussed with respect to the standard pattern of endto-end association in the Oregon- $\mathrm{R}$ wild-type stock that provided the control preparations. In the 4-2L rearrangement, the interaction of chromosome 4 with the tip of $2 \mathrm{~L}$ ( $101 \mathrm{E}$ with $21 \mathrm{~A}$ ) led to pronounced puffing in subdivisions $21 \mathrm{~A}$ and $\mathrm{B}$, as was most strikingly manifested when the distal segments of $2 \mathrm{~L}$ failed to synapse and the homologue of paternal origin showed a large puff whereas that of maternal origin (not carrying the fourth chromosome) remained unpuffed.
\end{abstract}

\section{Introduction}

Among a series of induced chromosomal rearrangements detected by cytological examination of salivary-gland chromosomes from the third-instar larval progeny of X-ray irradiated fathers and unirradiated mothers of the Oregon-R wild-type stock of Drosophila melanogaster, we found one reciprocal translocation that involved chromosome 4 and the "tip" of the left limb of the second chromosome (2L) and another of similar type that involved 4 and the "tip" of $2 \mathrm{R}$. In the $4-2 \mathrm{~L}$ rearrangement, the regions of breakage and reunion were determined to be in $101 \mathrm{E}$ and $2 \mathrm{IA}$; in the $4-2 \mathrm{R}$ rearrangement the points of exchange were found to be in $102 \mathrm{C}$ and $60 \mathrm{E}$. When the homologues that form the left or right limbs of chromosome 2 in these translocation heterozygotes are completely synapsed, as shown in Figs. 1 and 2, it is evident that 2L.4 is slightly Ionger than the normal 2L pairing partner of maternal origin,

* This study was supported in part by a Research Grant (GM-10499) from the National Institutes of Health, U.S. Public Health Service. 

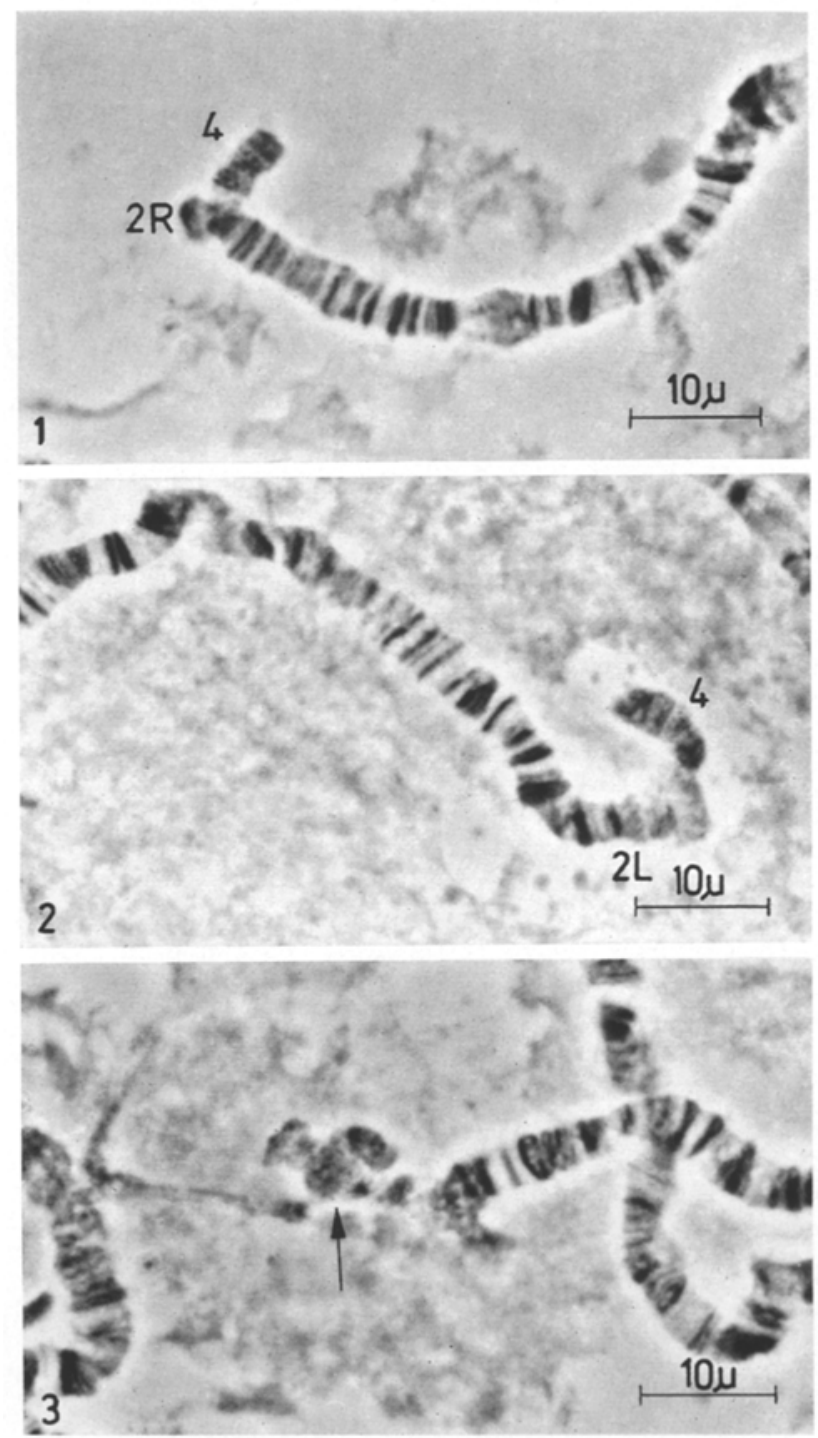

Fig. 1. The $2 \mathrm{R} \cdot 4$ part of an X-ray-induced reciprocal translocation, paternal (o) in origin, with tip of attached chromosome 4 extending beyond the $2 R$ strand of maternal ( 9 ) origin. Compare with Fig. 3

Fig. 2. The 2L.4 part of an X-ray-induced reciprocal translocation with tip of attached chromosome 4 projecting beyond $2 \mathrm{~L}$ 우

Fig. 3. Arrow indicates the $4 \cdot 2 \mathrm{R}$ part of a reciprocal translocation. $4 \cdot 2 \mathrm{R}$ is paired with base of normal 4 to give a $4 / 4 \cdot 2 \mathrm{R}$ configuration. (For the reciprocal type, 2R/2R $\cdot 4$, see Fig. 1) 
and that $2 \mathrm{R} \cdot 4$ is longer than its $2 \mathrm{R}$ partner. Lengthening of $2 \mathrm{~L}$ or $2 \mathrm{R}$ by addition of a section of chromosome 4 offers the prospect of determining the potential of this small chromosome to pair end-to-end with the limbs of the longer autosomes and the $\mathrm{X}(4$ with $2 \mathrm{R}, 3 \mathrm{~L}, 3 \mathrm{R}$ and $\mathrm{X}$ in one case; or 4 with $2 \mathrm{~L}, 3 \mathrm{~L}, 3 \mathrm{R}$, and $\mathrm{X}$ in the other). This prospect has been explored and the frequencies of endwise associations determined, as specified below. It should be noted that the complementary types $(4 \cdot 2 \mathrm{~L}$ and $4 \cdot 2 \mathrm{R})$ are tiny structures, since they consist only of the short left limb, centromere, and base of the right limb of chromosome 4 capped by either the tip of $2 \mathrm{~L}$ or $2 \mathrm{R}$. They can be identified in some nuclei where they are positioned in the chromocentral region as a consequence of pairing with the normal intact chromosome 4 of maternal origin (Fig. 3); but they rarely participate (in contrast with the $2 \mathrm{~L} \cdot 4$ and $2 \mathrm{R} \cdot 4$ types) in establishing end-to-end contacts with the non-homologous chromosome limbs. In some cells of the pair of glands carrying the 4-2L translocation, the $4 \cdot 2 \mathrm{~L}$ reciprocal type is not discernible and seems to have been eliminated; these cells contain in addition to the $2 \mathrm{~L} \cdot 4$ rearrangement, two intact synapsed normal fourth chromosomes (presumably of maternal origin and representing a non-disjunctional event during development). The glands themselves are accordingly mosaic, since other cells contain both the $4 \cdot 2 \mathrm{~L}$ and $2 \mathrm{~L} \cdot 4$ reciprocal types.

The end-to-end adhesion of the longer salivary-gland chromosomes of Drosophila was first reported by Bauer (1936) and ProkofyevaBelgovskaya (1937). A subsequent quantitative study - initiated in our laboratory - of the frequency of union in pairs among the five long limbs $(2 \mathrm{~L}, 2 \mathrm{R}, 3 \mathrm{~L}, 3 \mathrm{R}, \mathrm{X})$ in the Oregon-R wild-type stock of Drosophila melanogaster suggested that adhesions are not produced with the frequencies that would be expected if association occurred strictly at random (Hinton and Sparrow, 1941). More extensive studies by HinTON and ATwood (1941) and Hrrton (1945) confirmed the existence of a nonrandom pattern of specificity within a given stock, which differed from that of other stocks and apparently was subject to change with the passage of time. It should be emphasized that various lines of evidence, including in vitro studies of unfixed cells as well as micrurgical and other manipulative treatments of fixed chromosomes, indicate that terminal adhesions occur in the living cell and are not merely artifacts induced in the process of spreading the giant chromosomes for microscopical examination.

The 4-2L and 4-2R rearrangements exist as "one-of-a-kind" preparations, since sacrificing the $F_{1}$ larvae for study of the salivary-gland chromosomes eliminated the possibility that stocks could be established for extensive confirmatory analyses. It was necessary, therefore, to examine critically and record every diagnosable chromosome complex on each of the two slides. It was also necessary to score an adequate number of controls for each of the two rearrange- 
ments, since they were detected in different experiments separated in time by a period of about five months. This requirement was met by examining well-spread chromosome complexes from a series of normal preparations (showing no X-rayinduced aberrations) obtained from the same experiments as those that provided the $4-2 \mathrm{~L}$ and the $4-2 \mathrm{R}$ reciprocal translocations.

Culture bottles were kept at $17^{\circ} \mathrm{C}$ during the period of development of the larvae. Salivary glands, excised from third-instar larvae, were stained and squashed in aceto-carmine or acetic orcein and then processed by the method described by BRIDGES (1936).

The photomicrographs shown as Figs. 1 to 8 were prepared from negatives obtained by using the phase-contrast optical system of a Zeiss Photomicroscope.

\section{Results}

The frequencies of end-to-end associations involving tips of chromosomes in the 4-2L and 4-2R reciprocal translocations are given in Tables 1 and 2. It is obvious at first glance that the tip of the fourth chromosome plays in each case a significant role in the process of establishing end-to-end adhesions. Before attempting to assess those data in detail, attention will be directed to the "controls" listed in Tables 3 and 4, since the observations recorded there serve to define the patterns of endto-end adhesion in the Oregon- $R$ stock at the time the two different experiments were initiated. These tables include, in addition to the endto-end types of association, a few cases of adhesion of chromosomal tips to chromocentral materials. We have not scored the number of adhesions of chromosomal tips to intercalary heterochromatic regions, since precise data covering the frequency of such contacts would be difficult to assemble - as is evident from the study of ectopic pairing made by KAUF MANN and IDDLES (1965) — and only marginally related to the main topic of this paper.

The major difference between Tables 3 and 4 is in the proportion of cells that do not reveal any end-to-end adhesions among the tips of the longer chromosomes (the tips of $\mathrm{X}, 2 \mathrm{~L}, 2 \mathrm{R}, 3 \mathrm{~L}$, and $3 \mathrm{R}$ accordingly lie free). About two-thirds of the 416 nuclei examined in compiling the data for Table 3 fell into that category as compared with about one-half of the 400 nuclei listed in Table 4 . The conditions responsible for this difference remain unknown; we can only suggest that in our opinion it is not determined primarily by the quality of the preparations used or by variations in the scanning procedures. This opinion seems sustained by the observation that among the cells in which adhesions are recognizable, the frequencies of the different types are remarkably similar in the two sets of data. Stated somewhat differently, one might assume that when conditions are established that favor production of end-to-end contacts within a cell the different chromosomal limbs tend to adhere with frequencies that are characteristic of the stock involved. 
Table 1. End-to-end and other patterns of association of chromosome limbs detected in salivary-gland cells of Drosophila melanogaster larvae. $2 L$ carries the distal portion of 4 as the result of an X-rayinduced reciprocal translocation. In the table $2 L-4-X$ reters to the adhesion of the tip of 4 carried on $2 L$ to the tip of the $X$ chromosome. $2 R-3 R$ refers to the endto-end attachment of $2 R$ and $3 R .2 L^{\mathrm{ubm}}=$ unsynapsed homologue of maternal origin; $q=$ strand of maternal origin; chr. = chromocentral material

\begin{tabular}{ll}
\hline Patterns of association & $\begin{array}{l}\text { No. and } \\
\text { frequency }\end{array}$ \\
\hline
\end{tabular}

\begin{tabular}{lr}
$2 \mathrm{~L} \cdot 4-\mathrm{X}$ (see Fig. 5 ) & 23 \\
$2 \mathrm{~L} \cdot 4-2 \mathrm{~L}$ \% & 5 \\
$2 \mathrm{~L} \cdot 4-3 \mathrm{~L}$ & 1 \\
$2 \mathrm{~L} \cdot 4-3 \mathrm{~L} ; 2 \mathrm{R}-3 \mathrm{R}$ & 1 \\
$2 \mathrm{~L} \cdot 4-3 \mathrm{R}$ & 1 \\
$2 \mathrm{R}-3 \mathrm{R}$ & 1 \\
$3 \mathrm{~L}-3 \mathrm{R}$ & 1 \\
$2 \mathrm{~L} / 2 \mathrm{~L} \cdot 4$ and $4 / 4 \cdot 2 \mathrm{~L}$ synapse & 3 \\
2L.4-chr. 3L & 2 \\
2Luhm-chr. 3L & 1 \\
$\mathrm{X}$-chr. X & 2 \\
\hline Total & 41 \\
\hline
\end{tabular}

Ends of $\mathrm{X}, 2 \mathrm{~L} / 2 \mathrm{~L} \cdot 4,2 \mathrm{R}$, $3 \mathrm{~L}, 3 \mathrm{R}$ all lie free 59

Grand Total 100

Table 2. Contacts as defined in Table 1. In this case $2 R$ carries the distal part of 4 (a $2 R-4$ reciprocal translocation). $2 R \cdot 4$ $X-3 L-3 R$ describes a situation where the tip of 4 on $2 R$ adheres to the tip of $X$, as does the tip of $3 L$, to which the tip of $3 R$ adheres. ${ }^{u h}=$ unsynapsed homologue

\begin{tabular}{ll}
\hline Patterns of association & $\begin{array}{l}\text { No. and } \\
\text { frequency }\end{array}$ \\
\hline
\end{tabular}

\begin{tabular}{|c|c|}
\hline 2R.4-X (see Fig. 4) & 58 \\
\hline $2 \mathrm{R} \cdot 4-\mathrm{X} ; 2 \mathrm{R}-3 \mathrm{R}$ & 3 \\
\hline $2 \mathrm{R} \cdot 4-\mathrm{X} ; 3 \mathrm{~L}-3 \mathrm{R}$ & 1 \\
\hline $2 \mathrm{R} \cdot 4-\mathrm{X}-2 \mathrm{R}$ & 1 \\
\hline $2 \mathrm{R} \cdot 4-\mathrm{X}-3 \mathrm{~L}$ & 10 \\
\hline $2 R \cdot 4-X-3 L-3 R$ & 2 \\
\hline $2 \mathrm{R} \cdot 4-\mathrm{X}^{\mathrm{uh}}$; other $\mathrm{X}^{\mathrm{uh}}-3 \mathrm{~L}-2 \mathrm{R}$ & 21 \\
\hline 2R·4-3L (e.g., Fig. 6) & 4 \\
\hline $2 \mathrm{R} \cdot 4-3 \mathrm{R} ; 2 \mathrm{R}-3 \mathrm{~L}$ & 1 \\
\hline $2 \mathrm{R} \cdot 4-3 \mathrm{R}-2 \mathrm{R}$ & 2 \\
\hline $2 \mathrm{R}^{\mathrm{uh}} \cdot 4-4$ 우 & 2 \\
\hline $\mathrm{X}-2 \mathrm{~L}$ & 1 \\
\hline $\mathrm{X}-2 \mathrm{~L} ; 3 \mathrm{~L}-3 \mathrm{R}$ & 1 \\
\hline $\mathrm{X}-2 \mathrm{R}$ (but 4 on $2 \mathrm{R}$ lies free) & 1 \\
\hline $\mathrm{X}-3 \mathrm{~L}$ & 5 \\
\hline $\mathrm{X}^{\text {uh }}-3 \mathrm{~L}$; other $\mathrm{X}^{\mathrm{uh}}-2 \mathrm{~L}$ & I \\
\hline $\mathrm{X}-4$ q & 2 \\
\hline $3 \mathrm{~L}-2 \mathrm{R}$ (but 4 on $2 \mathrm{R}$ lies free) & 1 \\
\hline $3 \mathrm{~L}-3 \mathrm{R}$ & 1. \\
\hline $3 \mathrm{R}-2 \mathrm{R}$ (but 4 on $2 \mathrm{R}$ lies free) & 2 \\
\hline $2 \mathrm{R} / 2 \mathrm{R} \cdot 4$ and $4 / 4 \cdot 2 \mathrm{R}$ synapse & e 9 \\
\hline $\mathrm{X}$-chr. X & 3 \\
\hline Total & $\begin{array}{l}112 \\
72.3 \%\end{array}$ \\
\hline $\begin{array}{l}\text { Ends of } \mathrm{X}, 2 \mathrm{~L}, 2 \mathrm{R} / 2 \mathrm{R} \cdot 4 \\
3 \mathrm{~L}, 3 \mathrm{R} \text { all lie free }\end{array}$ & $\begin{array}{l}43 \\
27.7 \%\end{array}$ \\
\hline Grand Total & $\begin{array}{l}155 \\
100.0 \%\end{array}$ \\
\hline
\end{tabular}

The data in Tables 3 and 4 have been compared with respect to the frequencies of adhesion of ends in pairs (the method used by Hinton, Sparrow, and ATwood in their studies, which omits the more complex associations in groups of three or four), and with respect to the frequencies of involvement of the tips of individual chromosomes in all the recorded associations. In the first of these approaches, when the $\mathrm{X}-2 \mathrm{~L}$, X-2R, X-3L, X-3R, 2L-2R, 2L-3L, 2L-3R, 2R-3L, 2R-3R, and 3L-3R 
Table 3. "Control data" for Table 1. See that table for explanation of method of recording

\begin{tabular}{lc}
\hline Patterns of association & $\begin{array}{c}\text { No. and } \\
\text { frequency }\end{array}$ \\
\hline X-2L & 16 \\
X-2R & 4 \\
X-3L & 41 \\
X-3R & 15 \\
X-4 & 2 \\
X-2L; 2R-3R & 2 \\
X-2R; 2L-3L & 1 \\
2L-2R & 2 \\
2L-3L. & 1 \\
2L-3R & 2 \\
2R-3L & 5 \\
2R-3R & 14 \\
3L-3R & 10 \\
3L-4 & 1 \\
3L-X-3R & 3 \\
3L, 3R, 2L tips adhere & 1 \\
X-chr. X & 4 \\
2L-chr. 2L & 1 \\
2R-chr. 2R & 1 \\
3L-chr. 3L & 1 \\
3L-chr. X & 1 \\
3R-chr. 3R & 1 \\
Thtal & 129 \\
& $31.0 \%$ \\
\hline Ends of X, 2L, 2R, 3L, & 287 \\
and 3R lie free & $69.0 \%$ \\
\hline Grand Total & 416 \\
& $100.0 \%$ \\
\hline
\end{tabular}

Table 4. "Control data" for those of Table 2

\begin{tabular}{|c|c|}
\hline Patterns of association & $\begin{array}{l}\text { No. and } \\
\text { frequency }\end{array}$ \\
\hline $\mathrm{X}-2 \mathrm{~L}$ & 26 \\
\hline $\mathrm{X}-2 \mathrm{R}$ & 3 \\
\hline $\mathrm{X}-3 \mathrm{~L}$ & 58 \\
\hline X-3R & 25 \\
\hline$X-4$ & 2 \\
\hline $\mathrm{X}-2 \mathrm{~L} ; 2 \mathrm{R}-3 \mathrm{~L}$ & 1 \\
\hline $\mathrm{X}-3 \mathrm{~L} ; 2 \mathrm{R}-3 \mathrm{R}$ & 4 \\
\hline $\mathrm{X}-3 \mathrm{~L} ; 2 \mathrm{R}$-chr. $2 \mathrm{R}$ & 1 \\
\hline $\mathrm{X}-3 \mathrm{R} ; 2 \mathrm{~L}-2 \mathrm{R}$ & 1 \\
\hline $\mathrm{X}-3 \mathrm{~L}-2 \mathrm{R}$ & 2 \\
\hline X-3L-2L & 1 \\
\hline $\mathrm{X}-3 \mathrm{~L}-3 \mathrm{R}$ & 2 \\
\hline $\mathrm{X}-3 \mathrm{R}-3 \mathrm{~L}$ & 1 \\
\hline $\mathrm{X}, 2 \mathrm{R}, 3 \mathrm{R}$ tips adhere & 1 \\
\hline $\begin{array}{l}\mathrm{X}, 2 \mathrm{R}, 3 \mathrm{~L}, 3 \mathrm{R} \text { adhere } \\
\text { (Fig. 7) }\end{array}$ & 1 \\
\hline $2 \mathrm{~L}-3 \mathrm{R}$ & 2 \\
\hline 2L-3L; $X^{\text {uh }}$ chr. X & 1 \\
\hline 2L-3L; 2R-X-3R & I \\
\hline $2 \mathrm{~L}-\mathrm{X}-3 \mathrm{~L}$ & 1 \\
\hline $2 \mathrm{~L}-\mathrm{X}-3 \mathrm{R}$ & 1 \\
\hline $2 \mathrm{~L}-2 \mathrm{R}-3 \mathrm{R}$ & 1 \\
\hline $2 \mathrm{~L}-3 \mathrm{~L}-2 \mathrm{R}$ & 1 \\
\hline 2R-3L & 9 \\
\hline $2 \mathrm{R}-3 \mathrm{R}$ & 19 \\
\hline $3 \mathrm{~L}-3 \mathrm{R}$ & 13 \\
\hline $3 \mathrm{~L}-4$ & 1 \\
\hline $3 \mathrm{~L}-4-3 \mathrm{R}$ & 6 \\
\hline $3 \mathrm{R}-4$ & $\mathrm{I}$ \\
\hline$X$-chr. X & 5 \\
\hline 3L-chr. 3L & 1 \\
\hline 3R-chr. 3R; X-chr. 3L & 1 \\
\hline Total & $\begin{array}{l}193 \\
48.3 \%\end{array}$ \\
\hline $\begin{array}{c}\text { Ends of } \mathrm{X}, 2 \mathrm{~L}, 2 \mathrm{R}, 3 \mathrm{~L} \\
\text { and } 3 \mathrm{R} \text { all lie free }\end{array}$ & $\begin{array}{l}207 \\
51.7 \%\end{array}$ \\
\hline Grand Total & $\begin{array}{l}400 \\
100.0 \%\end{array}$ \\
\hline
\end{tabular}

classes in Table 3 are compared with those in Table 4 , using the $\chi^{2}$ method (some grouping being required because of the small numbers of the X-2R, 2L-2R, 2L-3L, and 2L-3R types), the $\chi^{2}$ obtained was 
3.48 for six degrees of freedom, which gave a $\mathrm{P}$ value close to 0.750 . In the other approach, in which all the adhesions of tips recorded in Tables 3 and 4 were compared, a slightly higher value of $\mathrm{P}$ was determined (Table 5). Both results indicate that any differences between the distributions specified in Tables 3 and 4 can be attributed to the sampling error.

Table 5. Comparison by the $\chi^{2}$ method of the frequencies, given in Tables 3 and 4 , with which tips of different chromosomes participate in end-to-end and other types of association. The 454 ends recorded in Table 4 are compared with values expected on the basis of the distribution of 274 ends reported in Table 3

\begin{tabular}{lrrrrrr}
\hline & \multicolumn{1}{c}{$\mathrm{X}$} & \multicolumn{1}{c}{$2 \mathrm{~L}$} & \multicolumn{1}{c}{$2 \mathrm{R}$} & \multicolumn{1}{l}{$3 \mathrm{~L}$} & \multicolumn{1}{c}{$3 \mathrm{R}$} & \multicolumn{1}{c}{ 4 and chrom. } \\
\hline Observed & 157.000 & 37.000 & 49.000 & 113.000 & 80.000 & \multicolumn{1}{c}{$18.000=454$} \\
Expected & 150.728 & $\mathbf{4 4 . 4 9 2}$ & 48.124 & $\mathbf{1 0 9 . 4 1 4}$ & 81.266 & $19.976=454$ \\
Difference & 6.272 & $\mathbf{7 . 4 9 2}$ & 0.876 & 3.586 & 1.266 & \multicolumn{1}{l}{1.976} \\
$\mathrm{~d}^{2} / \mathrm{e}$ & 0.261 & 1.262 & 0.016 & 0.118 & \multicolumn{1}{c}{0.020} & \multicolumn{1}{c}{0.195} \\
\hline
\end{tabular}

$\chi^{2}=1.872$ for 5 d.f.; $P=$ ca. 0.85 .

Table 6. Comparison of frequencies (\%) of pairing ends among chromosome limbs in the 2L-4 and 2R-4 translocations, and in the controls

\begin{tabular}{llllrrrrl}
\hline & $\mathrm{X}$ & $2 \mathrm{~L}$ & $2 \mathrm{~L} \cdot 4$ & $2 \mathrm{R}$ & $2 \mathrm{R} \cdot 4$ & $3 \mathrm{~L}$ & $3 \mathrm{R}$ & 4 and chrom. \\
\hline Controls & $\mathbf{3 4 . 1}$ & 8.8 & & 10.7 & & 24.6 & 17.7 & 4.1 \\
4-2L Tr. & 32.1 & 7.7 & 42.3 & 2.6 & & 3.8 & 5.1 & 6.4 \\
4-2R Tr. & 40.7 & 1.2 & & 4.7 & 32.9 & 12.0 & 5.8 & 2.7 \\
\hline
\end{tabular}

Especially noteworthy in this analysis (Table 6 ) is the observation of a high frequency of participation in pairing of the tip of the $X(34,1 \%)$, followed by successively lower frequencies of $3 \mathrm{~L}(24.6 \%), 3 \mathrm{R}(17.7 \%)$, $2 \mathrm{R}(10.7 \%)$, 2L $(8.8 \%)$, and 4 and chromocentral associations $(4.1 \%)$. This distributional pattern, which was quite similar in the two samples collected in experiments separated in time by five months, does not resemble any of the patterns reported by Hinton, Sparkow, and ATwood in their studies of the Oregon-R stock. If we compare, for example, the first five items in our Table 5, namely, $\mathrm{X}=157,2 \mathrm{~L}=37,2 \mathrm{R}=49$, $3 \mathrm{~L}=113,3 \mathrm{R}=80$ (omitting 4 and chrom.$=18)$, with the values expected on the basis of Hinton and SPARRow's (1941) study, a $\chi^{2}$ value of 31.72 is obtained, which for four degrees of freedom gives a probability of less than 0.001 that the observed differences can be attributed to chance deviations.

In the Oregon- $\mathrm{R}$ stock that we have used, the tips of $2 \mathrm{~L}$ and $2 \mathrm{R}$ are relatively ineffective in establishing end-to-end contacts, since they 

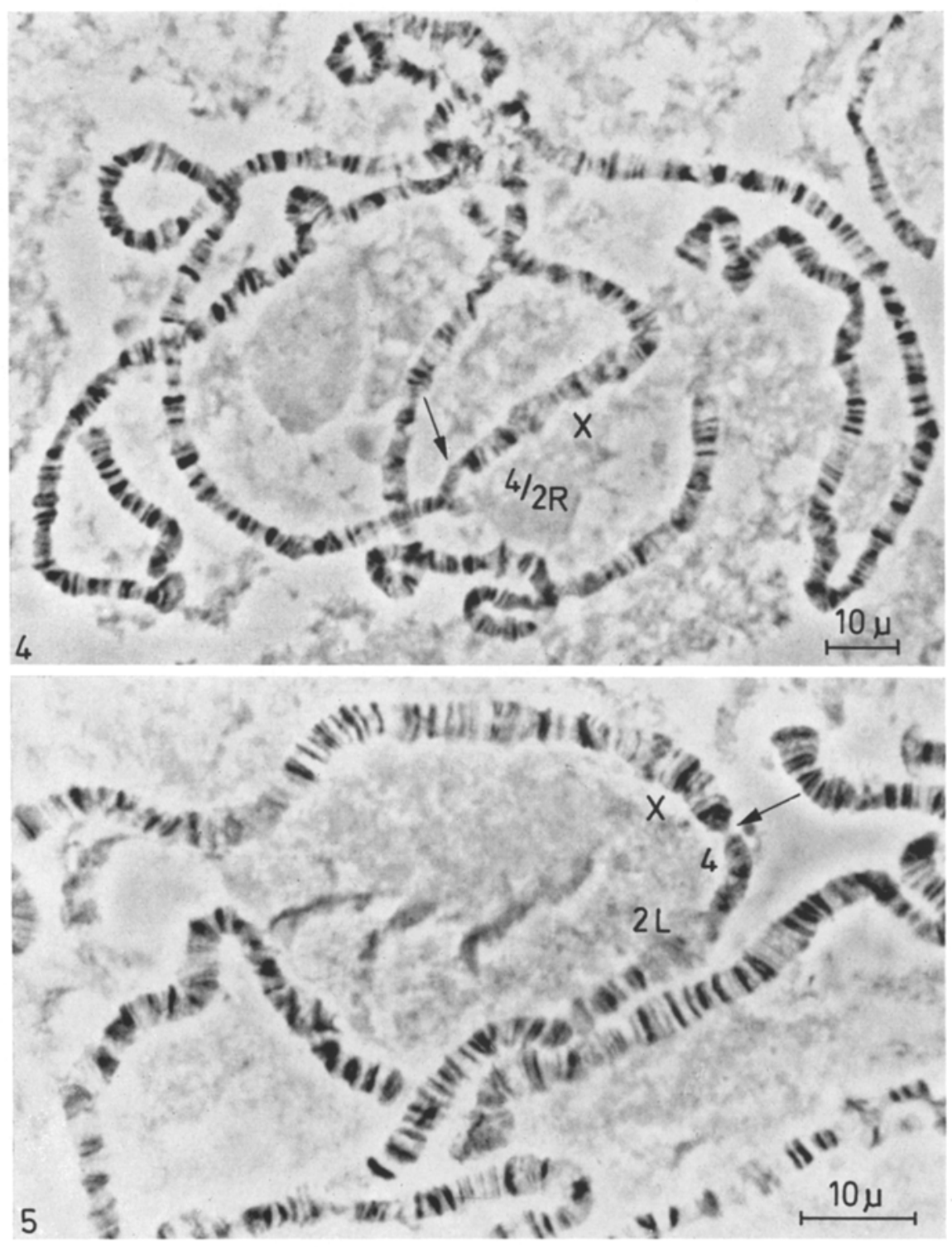

Fig. 4. End-to-end attachment of tip of $\mathrm{X}$ with tip of 4 carried on $2 \mathrm{R}$. Arrow indicates region of adhesion

Fig. 5. End-to-end attachment of tip of $\mathrm{X}$ with tip of 4 carried on $2 \mathrm{~L}$

provide respectively only about 9 and 11 per cent of the "pairing ends" (Table 6). But when capped by the distal end of the fourth chromosome, the $2 \mathrm{~L} .4$ chromosome of paternal origin supplies 42 per cent of the de- 

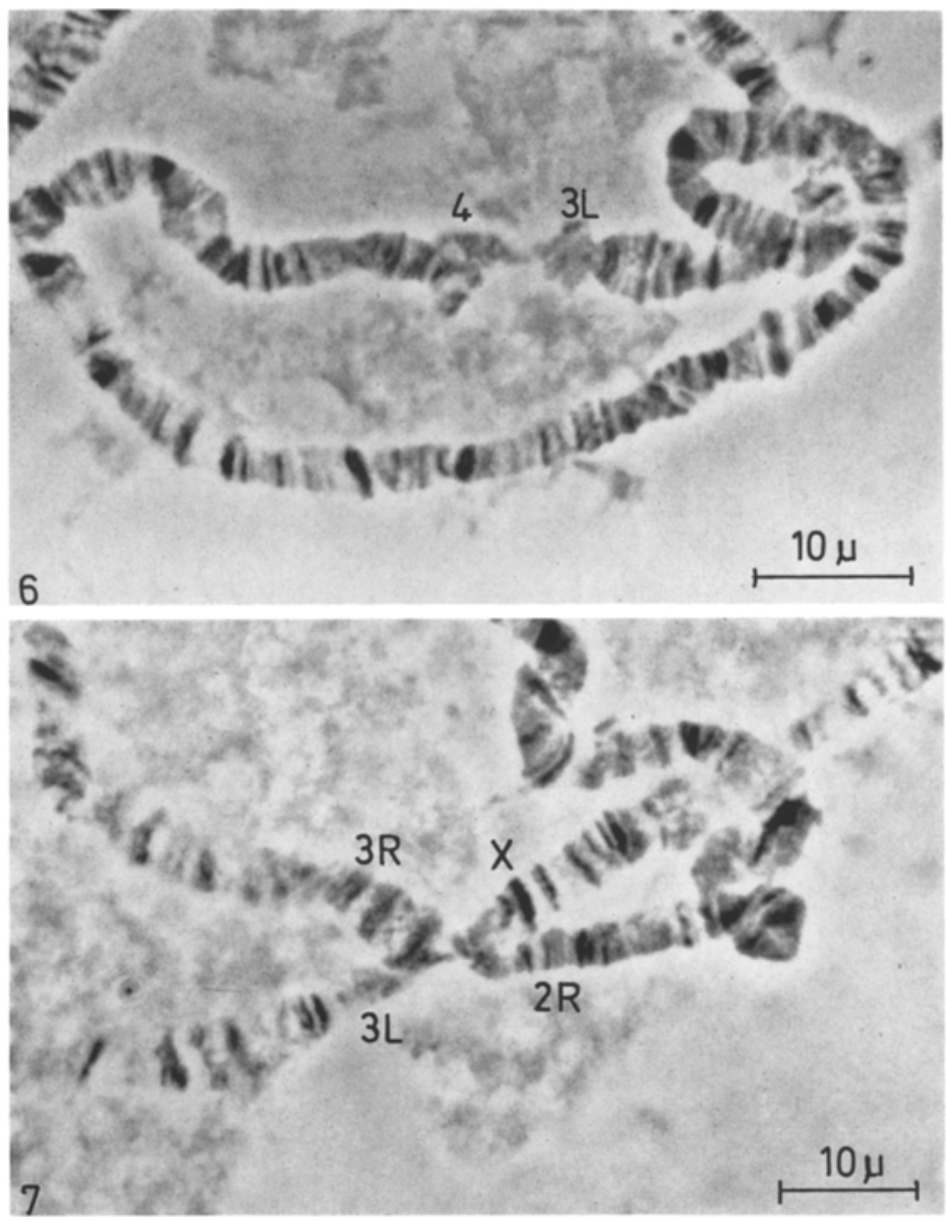

Fig. 6. End-to-end adhesion of tip of $3 \mathrm{~L}$ with tip of 4 carried on $2 \mathrm{R}$ Fig. 7. Mutual attachment of tips of $\mathrm{X}, 2 \mathrm{R}, 3 \mathrm{~L}$, and $3 \mathrm{R}$

tectable pairing ends, and $2 \mathrm{R} \cdot 4$ supplies 33 per cent. A high proportion of these contacts are with the $\mathrm{X}$ chromosome (ca. $59 \%$ of the total number in each of the two cases). As noted in Table 6, the over-all frequency of pairing of the tip of the $\mathrm{X}$ with the translocated fourth and the ends of other chromosomes of the complex is 32 per cent in the $2 \mathrm{~L} \cdot 4$ translocation, and 41 per cent in the $2 \mathrm{R} \cdot 4$ translocation. Since the value for the controls is 34 per cent, it is apparent that the pairing potential of the tip of chromosome 4 when it is attached to either $2 \mathrm{~L}$ or $2 \mathrm{R}$ is not expressed at the expense of the pairing ability of the tip of the $\mathrm{X}$ chromosome. Reduction of frequencies, as compared with those of the controls, must accordingly be reflected in the relative inactivity of the tips of $2 \mathrm{~L}, 2 \mathrm{R}$, 


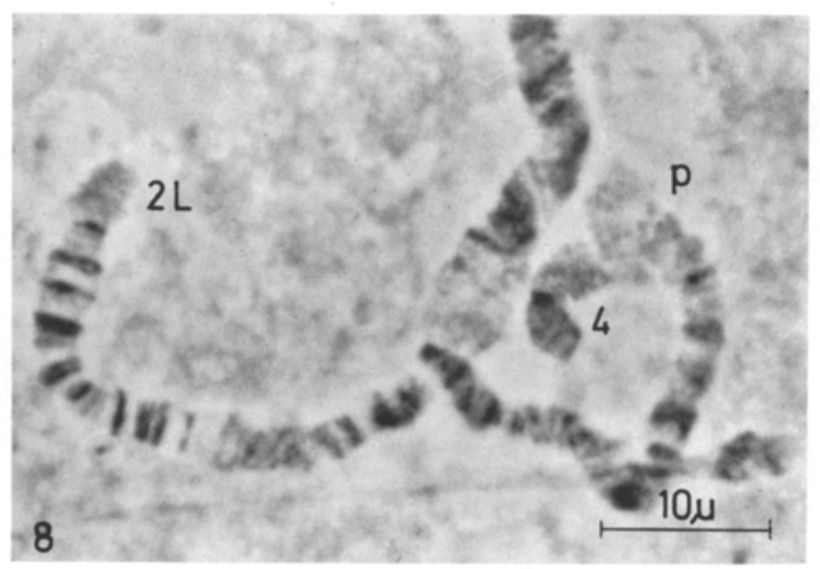

Fig. 8. Distal portions of $2 \mathrm{~L}$ and $2 \mathrm{~L} \cdot 4$ have not paired. A pronounced puff $(p)$ appears at tip of $2 \mathrm{~L}$ in $\overrightarrow{0}$ strand carrying chromosome 4 ; no puff of comparable magnitude is present in $2 \mathrm{~L}$ 우

$3 \mathrm{~L}, 3 \mathrm{R}$, and 4 at chromocenter. Inspection of Table 6 shows that $3 \mathrm{~L}$ and $3 R$ are much less active in pairing in the translocation heterozygotes than in the controls, whereas $2 \mathrm{~L}$ and $2 \mathrm{R}$ are somewhat less inhibited in the expression of their pairing potential as compared with the controls. The high frequency of participation of the 2L tip in the 2L-4 translocation ( $7.7 \%$ as compared with $8.8 \%$ in the controls) is attributable in large measure to five observed cases in which the tip of 4 attached to $2 \mathrm{~L}$ curved downwards and adhered to the tip of the synapsed normal $2 \mathrm{~L}$ strand of maternal origin. No comparable pattern of association was detected among the $4 \cdot 2 \mathrm{R} / 2 \mathrm{R}$ chromosome limbs examined, which may be due to the fact that the terminal section of 4 attached to $2 \mathrm{R}$ is considerably shorter than the section of 4 attached to $2 \mathrm{~L}$.

\section{Discussion}

Observations of the type reported in this paper offer promise of increasing our understanding of the conditions that determine the patterns of association among the chromosome ends in salivary-gland cells of Drosophila melanogaster. Some knowledge has now been gained about the pairing potential of the tip of chromosome 4 when positioned so that it is free to "compete" with the tips of the longer autosomes and the $X$ (Figs. 4,5). The two translocations studied, with a limited number of cells available for analysis, provide at best a crude estimate of this potential. An additional number of rearrangements, involving not only 4-2L and 4-2R, but also 4-3L, 4-3R, and 4-X, would be required to obtain the data essential for broadening the perspectives needed in 
evaluating pairing patterns with respect to the degree of homology that exists between the ends of the different chromosome limbs. But viable reciprocal translocations having one break at the base of chromosome 4 and the other in the most distal subdivision of one of the long chromosome limbs are difficult to obtain, even following treatment with heavy doses of $\mathrm{X}$ rays or other agents that induce chromosomal breakage. Rearrangements meeting these specifications have occasionally been detected by other workers; an example is $\mathrm{T}(1-4) \mathrm{Al} 0$, having breaks at $1 \mathrm{~A} 6$ and $102 \mathrm{Al}$ (according to STONE and GRIFren, 1940), but this seems to have been lost (LindsLey and GreLL, 1967), as have some others, so that they are no longer available for determining pairing patterns of their salivary-gland chromosomes.

Despite the limitations of the present study, certain aspects of the findings offer guide lines for future endeavors whenever supplementary rearrangements become available. In the first place, it is now apparent that a reliable base line for meaningful comparisons can readily be established by determining the standard pairing pattern within a given stock. Confidence about this possibility stems from the finding that the data collected in two separate experiments (recorded in Tables 3 and 4) are quite similar. The uniformity may be attributable, in part at least, to the scoring technique used, which involved the diagnosis of every available cell on a given slide, as compared with the selective-sampling method employed, for example, by Hinton and Sparrow, who noted that , adhesion may be observed roughly in one cell out of each two pairs of salivary glands." In the second place, the pairing patterns observed in the 4-2L and 4-2R translocations suggest that the affinity between the tip of the attached fourth chromosome and that of the $\mathrm{X}$ is strong (Figs. 4, 5). As mentioned previously, this type of adhesion accounts for about 59 per cent of the total number of associations in pairs. If, on the other hand, only rearrangements involving $2 \mathrm{~L} \cdot 4$ or $2 \mathrm{R} \cdot 4$ are considered the frequencies in the two cases are respectively 70 and 89 per cent. Obviously, the tips of 4 and $\mathrm{X}$ are capable of exhibiting strong mutual attractions. If this bespeaks structural similarity, the tip of 4 is much more like the tip of $\mathrm{X}$ than that of any other chromosome limb. Although the end of a chromosome is the important part in determining the types of adhesions in which it can participate, it may be visualized as a region containing several properties for sticking, including weak as well as strong affinities. If the strongest possible affinity cannot be realized, a weaker attraction determines the predominant type (HrNToN, 1945). Thus, when chromosome 4 is ,, isolated" from the pairing pool by virtue of its normal chromocentral position, the tip of $\mathrm{X}$ in our Oregon- $\mathrm{R}$ stock pairs preferentially with the tip of $3 \mathrm{~L}$, although none of the other possible combinations is necessarily excluded thereby (Tables 3 and 4). 
The $\mathrm{X}$ chromosome (or a limb of one of the other long chromosomes) may even double back in a circle, so that the tip is in contact with the tip of 4 or with some less readily defined chromocentral material (PRoKofrevaBELgovskayA, 1937, noted that a marked increase in this type of association occurs in structural rearrangements, such as $\mathrm{T}(1-4) \mathrm{w}^{{ }^{m}}{ }^{5}$, which bring the tip of the $\mathrm{X}$ close to the chromocenter).

Evidence for homology between the $\mathrm{X}$ and fourth chromosomes of Drosophila melanogaster was deduced by SANDLER and Novitski (1956) from the observation that primary non-disjunction is greater in triplofour females than in the normal diplo-fours. Assumedly, the extra fourth chromosome occasionally pairs with and causes non-disjunction of the $\mathrm{X}$ chromosomes. The authors suggest that the regions of shared homology are in the proximal heterochromatin and are responsible for the pattern of disjunction. Other genetic evidence of homology between the $\mathrm{X}$ and fourth chromosomes had been presented by Gershenson (1940), LindsLey and Novitski (1953), and PARKER (1954).

More recently, GreLl and Grell (1960) designed an experiment to determine whether regions of shared homology in the proximal heterochromatin are solely responsible for non-homologous associations among chromosomes during oogenesis. Two chromosomes - the fourth and a so-called T4 fragment that carried the centromere and proximal heterochromatin of the fourth chromosome up to $101 \mathrm{~F}$, where the essentially euchromatic part of $3 \mathrm{R}$ distal to $86 \mathrm{D}$ was attached - were tested for their frequency of association with the $\mathrm{Y}$ chromosome and a small grossly deleted $X$ chromosome that consisted only of the proximal heterochromatic region and the distal euchromatic tip. It was found that the $\mathrm{Y}$ and small $\mathrm{X}$ associate much more frequently with $\mathrm{T} 4$ than with chromosome 4, a preference that is attributed to the euchromatic constitution of the distal portion of the T4 chromosome.

The problem of how chromosomes recognize one another and associate in special ways has been examined in detail by RHoda GreLL (1967), who used genetic and cytogenetic criteria for identifying chromosomal interactions during oogenesis in Drosophila melanogaster. Two types of pairing in the course of meiosis are recognized, namely, exchange pairing that precedes exchange and distributive pairing that follows thereafter. It is concluded from experimental evidence that the initial pairing is parasynaptic and that recognition is correlated with the extent of euchromatic homology. Distributive pairing, on the contrary, is correlated with the total size of the chromosome, is independent of homology, and is restricted to chromosomes that have not undergone exchange with an independent homologue. GreLL recognizes that it has become increasingly satisfying to think of base complementarity as the source of pairing specificity, but questions whether the initial attraction 
that brings chromosomes into register is achieved at that level of interaction. Although her extensive series of experiments lend support to alternative explanations of the pairing phenomenon - in showing, for example, that an $\mathrm{X}$-chromosome duplication is as readily recognized by chromosome 4 as another fourth chromosome if it is of comparable length, irrespective of its heterochromatic or euchromatic constitution - the diagnostic criteria are genetical or cytogenetical and offer no direct measure of molecular interactions. Despite concerted efforts over many years, the details of meiosis in the female Drosophila still remain refractory to cytological and cytochemical analysis. In contrast, the giant salivary-gland chromosomes have been much more amenable to analysis by cytological, cytochemical, radioautographical, and electronmicroscopical methods. Problems of chromosome recognition can be studied at the level of association of homologues, at that of ectopic pairing: of intercalary regions (assumedly involving heterochromatin), and at the level of end-to-end association of non-homologous chromosome limbs (probably also involving heterochromatin). These are not necessarily mutually exclusive categories, since the tip of an unsynapsed homologue may pair with the tip of a given chromosome limb, whereas that of the other homologue may find a different pairing partner or remain unpaired.

With respect to functional properties, we found that the attachment of chromosome 4 to the tip of $2 \mathrm{~L}$ in the $4-2 \mathrm{~L}$ rearrangement led to puffing in subdivisions $21 \mathrm{~A}$ and $\mathrm{B}$, a phenomenon that is most strikingly manifested in those cases where synapsis of $2 \mathrm{~L}$ fails and the homologue of maternal origin remains unpuffed (Fig. 8). Some measure of gene action should be obtainable in such cases by radioautographical and cytochemical methods. Since puffing that involves the synthesis of RNA can be measured in excised salivary-gland cells by the incorporation of, say, tritiated uridine, it should be possible to determine experimentally the nature of the interaction between two loci - such as $101 \mathrm{E}$ and $21 \mathrm{~A}$ in the 2L-4 translocation heterozygote - whenever suitable rearrangements become available for extended studies. To what extent heterochromatic or euchromatic materials are involved in such interactions - and in the establishment of specific end-to-end contacts - should also be determinable cytologically following pulse labeling with tritiated thymidine, as specified by Gay (1963). (Biochemical studies by Perreault, Gay and KaUfmanN, 1966, 1968, of DNA extracted from Drosophila suggest that heterochromatin and euchromatin do not differ significantly with respect to base composition). We thus find ourselves in the position of delineating the scope of a problem and suggesting methods for its resolution while attempting to garner the chromosomal rearrangements requisite for the task. 
Only after the foregoing part of this manuscript was written did we see the interpretation of Berendes and MEYeR (1968), derived from electron-microscopical studies, that end-to-end association of the long chromosomes in Drosophila hyde $i$ involves the fusion of compact terminal structures, which are assumed to be submicroscopic manifestations of the so-called telomeres. Our efforts with electron-microscopical and radioautographical techniques have been directed toward an understanding of the extent to which patterns of pairing involving the tips of two chromosome limbs in the Oregon-R stock of Drosophila melanogaster differ from those patterns that involve the tip of one chromosome and heterochromatic material located elsewhere among the chromosomes (in the chromocentral or intercalary regions). The findings and a discussion of the problem will be given in a subsequent publication.

\section{Literature}

BAUER, H.: Structure and arrangement of salivary gland chromosomes in Drosophila species. Proc. nat. Acad. Sci. (Wash.) 22, 216 - 222 (1936).

Berendes, H. D., and G. F. Meyer: A specific chromosome element, the telomere of Drosophila polytene chromosomes. Chromosoma (Berl.) 25, 184-197 (1968).

BRIDGES, C. B.: Current methods for permanent aceto-carmine smears. Drosophila Inform. Serv. 6, 31-35 (1936).

GaY, HeLEn: Chromosome structure and function. Carnegie Inst. Wash. Year Book 62, $503-510(1963)$.

Gershenson, S.: The nature of the so-called genetically inert parts of chromosomes. Vid. Akad. Nauk U.R.R.S. 3, 116 (Ukrainian with English summary), (1940).

Greld, Rhoda F.: Pairing at the chromosome level. J. cell. Physiol. 70, Suppl. 1, $119-145$ (1967).

-, and E. H. GRELL: The behavior of nonhomologous chromosomal elements involved in nonrandom assortment in Drosophila melanogaster. Proc. nat. Acad. Sci. (Wash.) 46, 51-57 (1960).

HrNton, T.: A study of chromosome ends in salivary gland nuclei of Drosophila. Biol. Bull. 88, $144-165$ (1945).

—, and K. C. Atwoon: Terminal adhesions of salivary gland chromosomes in Drosophila. Proc. nat. Acad. Sci. (Wash.) 27, 491-496 (1941).

-, and A. H. SparRow: The non-random occurrence of terminal adhesion in salivary chromosomes of Drosophila (Abstract). Genetics 26, 155 (1941).

Kaufmann, B. P., and M. K. IDdLes: Ectopic pairing in salivary-gland chromosomes of Drosophila melanogaster. Port. Acta biol. A 7, 225-248 (1965).

LindSley, D. L., and E. H. GReLd: Genetic variations of Drosophila melanogaster. Carnegie Inst. Wash. Publ. No. 627 (1967).

-, and E. Novitski: Frequent linkage of $\mathrm{Y}^{\mathrm{S} X} . \mathrm{Y}^{\mathrm{L}}$ and chromosome 4. Drosophila Inform. Serv. 27, 99 (1953).

Parker, D. R.: Radiation induced exchanges in Drosophila females. Proc. nat. Acad. Sci. (Wash.) 40, 795-800 (1954). 
Perreault, W. J., H. Gay, and B. P. Kaufmann: Base composition of DNA in heterochromatin of Drosophila melanogaster. Drosophila Inform. Serv. 41, 80 (1966).

- B.P. KaUfmann, and H. GAY: Similarity in base composition of heterochromatic and euchromatic DNA in Drosophila melanogaster. Genetics 60, $289-301$ (1968).

Prokofyeva-Belgovskaya, A. A.: Inert regions in the distal ends of chromosomes of Drosophila melanogaster. Bull. Acad. Sci. U.R.S.S. (ser. Biol.) 3, 719-724 (1937).

SANDLER, L., and E. Novitski: Eyidence for genetic homology between chromosomes I and IV in Drosophila melanogaster, with a proposed explanation for the crowding effect in triploids. Genetics 41, 189-193 (1956).

Stone, W. S., and A. B. Griffen: Changing the structure of the genome in Drosophila melanogaster. Univ. Texas Publ. 4082, 208-217 (1940).

Prof. Dr. B. P. Kaufmann, Dr. Helen Gay

Department of Zoology and Cytogenetics Laboratory

of Carnegie Institution of Washington

University of Michigan, Ann Arbor, Michigan 48104, U.S.A. 